\title{
Mullins thresholds in context of the network alteration theories
}

\author{
Hesam Khajehsaeid \\ School of Engineering-Emerging Technologies, University of Tabriz, Tabriz, Iran
}

\section{A R T I C L E I N F O}

\section{Keywords:}

Mullins softening

Rubber

Evolution law

Network alteration theory

\begin{abstract}
A B S T R A C T
Rubbers undergo a softening phenomenon in first cycles of loading where it is known this softening is more pronounced in filled rubbers. Existence of a clear physical interpretation for this phenomenon called Mullins effect is of great importance in development of constitutive relations and numerical simulations. In this paper, a recently proposed network alteration theory is extended to obtain a general form of evolution laws based on the physical description of the material molecular network. The resulting evolution laws are defined based on different measures to evaluate efficiency and accuracy of each measure in predicting the Mullins softening in context of the network alteration theories. Two experimental data sets on natural rubber are thoroughly investigated to determine a reliable threshold for this effect. It is concluded that, the maximum principal stretch and the Euler-Almansi strain are not good thresholds for Mullins effect at all. Other investigated measures are relatively efficient at small stretches but they mostly become less and less accurate at large stretches. However, the stored strain energy preserves its accuracy at the whole stretch range.
\end{abstract}

\section{Introduction}

Rubbers exhibit a nonlinear mechanical behavior including hysteresis, viscoelasticity and also a softening in first cycles of loading. Though this softening phenomenon called Mullins effect has also been reported for unfilled rubbers, but it is more substantial in filled rubbers $[1,2]$. In recent decades, the subject has been extensively studied by many authors from several viewpoints; however, there is still no clear interpretation for the physical origin of this phenomenon. Existence of a clear physical interpretation would be so beneficial for characterizing the evolution of material molecular network as well as numerical simulations [3-7] and design purposes.

A classical approach is the continuum damage mechanics (CDM) which assigns a modified strain energy function for the softened material using a damage parameter [8]:

$W=(1-d) W_{0}$

where $d$ is the damage parameter and $W_{0}$ is the strain energy density of the virgin material. Many constitutive equations have been proposed in this framework [9-12] where they mainly differ in definition of the damage criteria and/or the damage evolution laws. Laiarinandrasana et al. [13] have utilized maximum principal stretch where Lion [14] uses the Piola strain as the damage criteria. Also, some authors suggest the amount of stored strain energy as the damage parameter for constitutive equations [15-17]. In this context, Machado et al. [18] have experimentally investigated efficiency of different damage parameters for filled silicone rubber.
An alternative approach goes through the physical theories. These theories focus on qualitative description of microstructural alterations in material molecular network [19-21]. Inserting these theories in equations has led to macromolecular models [22-24]. Network alteration theories as a category of the macromolecular models are defined based on the alterations induced in material molecular network during deformation [25-27]. The network alteration theory of Marckmann [27] employs the idea of links breakage in molecular network without emphasis on nature of broken links. According to this theory, some chains and also weak molecular interactions cannot withstand the applied deformation and breakdown. Consequently, the number of junction points between chains decreases which raises the average length of chains $(N)$. On the other hand, number of the active chain segments per unit volume $(n)$ decreases due to these breakages. However, number of active monomer segments per unit volume $(n N)$ must remain constant to satisfy the principle of mass conservation. Khajehsaeid [25] showed that, results of this theory are not consistent when one uses different hyperelastic models to obtain evolution of the network. Comparing the bond energy of physical filler-chain interactions with bond energies of covalent cross-links (including monosulfidic, disulfidic and polysulfidic bonds), it was concluded that the weak physical bonds are more susceptible to break during deformation rather than the cross-links. Considering morphology of the filler-chain interactions and inspired by the Langmuir's theory, the adsorption and desorption of chains on fillers surface were formulated. Furthermore, it was shown that, in contrast to the previous theories, the number of

E-mail address: Khajehsaeid@Tabrizu.ac.ir. 
Table 1

Quantities used as driving evolution parameter.

\begin{tabular}{|c|c|c|}
\hline$\delta$ & Description & Refs. \\
\hline$I_{1}-3$ & $I_{1}=\operatorname{tr}(\mathbf{C}), \mathbf{C}=$ Right Cauchy - Green tensor & {$[25]$} \\
\hline$\sqrt{\text { B. B }}-\sqrt{3}$ & $\mathbf{B}=$ Left Cauchy - Green tensor & [33] \\
\hline$\lambda_{\max }-1$ & $\lambda_{\max }=$ Largest eigenvalue of deformation gradient $(\mathrm{F})$ & [13] \\
\hline $\operatorname{tr}\left(e^{2}\right)$ & Euler-Almansi strain: $\boldsymbol{e}=1 / 2\left(\mathbf{I}-\mathbf{B}^{-1}\right)$ & {$[34]$} \\
\hline $\operatorname{tr}\left(\epsilon^{2}\right)$ & Piola strain: $\boldsymbol{\epsilon}=1 / 2\left(\mathbf{C}^{-1}-\mathbf{I}\right)$ & [14] \\
\hline $\boldsymbol{W}$ & $W=$ Strain energy density & [15-17] \\
\hline
\end{tabular}

active monomers in the network is not necessarily a decreasing function of the deformation but it can even increase.

In this paper, the network alteration theory proposed by Khajehsaeid [25] is extended to obtain more general forms of evolution laws for rubbers molecular network. Rate of desorption of chains from fillers surface and consequently the arisen evolution laws are defined based on different measures to evaluate efficiency and accuracy of each measure in predicting the Mullins softening in context of the network alteration theories. Two experimental data sets in different strain ranges are thoroughly investigated to determine a reliable threshold of this effect for natural rubber.

\section{Generalized form of the theory}

Since there is still no unanimous interpretation for the physical origin of the Mullins effect, the mechanical quantity characterizing threshold of this effect has not been clearly defined. In attempt to provide a physical interpretation, the nature of broken links in material micro-structure has been noticed in [25] with focus on the role of filler particles and their interactions with molecular chains. A filler-chain bond is made if at least one monomer segment (or an end) of a chain is adsorbed on a filler surface. The other end of the chain may be free, adsorbed on another particle or linked to the chains network. Since there are a certain number of interaction positions on each filler particle, first chains contacting the surface will find more free positions. Formation of the first bond will increase the probability of adsorption of the neighboring segments. Consequently, the first chains make strong bonds but the later chains would find much less free positions and their bonds with filler particles would be weaker and easily removable by small tensions during deformation. After a while, a balance would be achieved between adsorption and desorption of segments on filler particles. However, applying a deformation to the material accelerates the breakage of weak physical filler-chain bonds and affects the balance. A Langmuir-type theory has been proposed for this phenomenon in [25]:

$\rho^{a} \varphi^{f}=\rho^{d} \varphi^{o}$

where $\rho^{a}$ and $\rho^{d}$ are the rates of chains adsorption and desorption on filler particles, respectively. Also $\varphi^{f}$ and $\varphi^{o}$ are the fractions of free and occupied interaction positions on the filler particles, respectively. Noting $\varphi^{f}+\varphi^{o}=1$, we have:

$\varphi^{o}=\frac{\rho^{a}}{\rho^{a}+\rho^{d}}$

Since the number of available chains nearby a free position is constant [28], $\rho^{a}$ is assumed to remain unchanged during deformation. However, the rate of desorption $\rho^{d}$ strongly depends on the amount of applied deformation:

$\rho^{d}=\rho^{d}(\delta)$

where $\delta$ is an arbitrary measure of deformation or any other damagelike parameter. In [25] it was assumed that the rate of desorption varies linearly during the deformation: $\rho^{d}=\rho_{0}{ }^{d}\left(1+A_{1} \delta\right)$

where $A_{1}$ is a positive constant and $\rho_{0}{ }^{d}$ is the value of the desorption rate at the initial balance state. Considering (2), (3) and (5) the fraction of occupied positions on filler particles is obtained:

$\varphi^{o}=\frac{\rho^{a}}{\rho^{a}+\rho_{0}{ }^{d}\left(1+A_{1} \delta\right)}=\frac{1}{A_{2}+A_{3} \delta}$

According to (6) the number of filler-chain interactions decreases with respect to $\delta$. This equation directly depends on the definition of the rate of desorption, i.e. (4). As one uses a m-th order or an exponential function for $\rho^{d}$, he/she obtains:

$\varphi^{o}=\frac{\rho^{a}}{\rho^{a}+\rho_{0}{ }^{d}\left(1+B_{1} \delta^{m}\right)}=\frac{1}{B_{2}+B_{3} \delta^{m}}$

or

$\varphi^{o}=\frac{\rho^{a}}{\rho^{a}+\rho_{0}{ }^{d} e^{C_{1} \delta}}=\frac{1}{1+C_{2} e^{C_{1} \delta}}$

The number of filler-chain interactions affects the chain density as a characteristic network parameter. When breakage of filler-chain bonds occurs, a chain may convert to a dangling one which leads to decrease in the number of (active) chains. Another possible phenomenon is detachment and activation of some chains which were asleep on fillers surface so far. In contrast to the former phenomenon, this case increases the number of active chains. Some chains may also be partly asleep on fillers. Breakage of filler-chain bonds can increase the active length of such chains and hence, the average chain length of the network. Chain density for a filled molecular network is calculated as below [29]:

$\mu=\left(1-\phi_{f}\right) \mu^{g u m}+\phi_{f} \mu^{f}\left(\varphi^{o}\right)$

where $\phi_{f}$ is the filler volume fraction, $\mu^{\text {gum }}$ corresponds to the chain density of the unfilled network and $\mu^{f}$ corresponds to the effect of filler-chain interactions $\left(\varphi^{o}\right)$ on the network cross-link density. Inserting (7) and (8) into (9) one obtains:

$\mu=\left(1-\phi_{f}\right) \mu^{\text {gum }}+\phi_{f} \frac{\mu_{0}^{f}}{1+\frac{B_{3}}{B_{2}} \delta^{m}}=\frac{1+B_{4} \delta^{m}}{B_{5}+B_{6} \delta^{m}}$

or

$\mu=\left(1-\phi_{f}\right) \mu^{\text {gum }}+\phi_{f} \frac{\mu_{0}{ }^{f}\left(1+C_{2}\right)}{1+C_{2} e^{C_{1} \delta}}=\frac{1+C_{3} e^{C_{1} \delta}}{C_{4}+C_{5} e^{C_{1} \delta}}$

where $\mu_{0}{ }^{f}$ is the initial value of $\mu^{f}$ at the undeformed state. It is noted that, the filler volume fraction (explicitly) and also its distribution pattern and particles size (implicitly embedded in $\mu_{0}{ }^{f}$ ) appear in the constitutive relations (10) and (11). Therefore, one can easily investigate the effects of these items on the material parameters of the proposed relations [30].

As noted by Khajehsaeid [25], the second consequence of (5) is dependency of the average length of chain segments on $\delta$. Average chain length of a filled network is calculated as follows: 

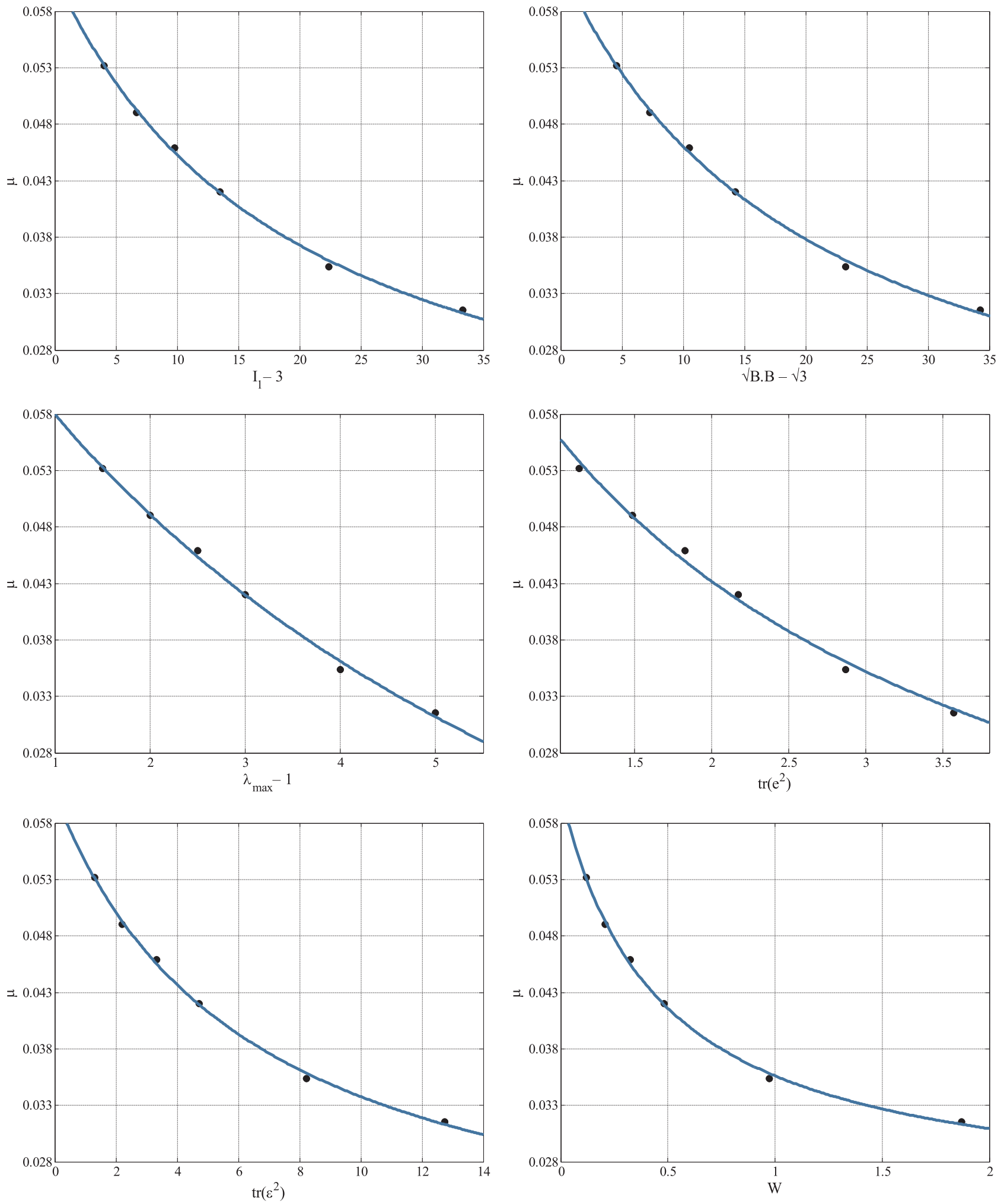

Fig. 1. Evolution of normalized network chain density during deformation for data reported in [32]. Lines: theory, marks: experimental values.

$N=\left(1-\phi_{f}\right) N^{g u m}+\phi_{f}\left(N_{f r e e}^{f}+N_{\text {detached }}^{f}\left(\varphi^{o}\right)\right)$

where $N^{g u m}$ is average chain length of the unfiled gum, $N^{f}$ free is average free length of the partly asleep chains on fillers and $N^{f}$ detached is average detached length of these chains. One may conclude $N^{f}$ detached is equal to the change in the average length of the sleeping parts. Length of the sleeping parts depends on the number of monomer segments lying down on the surface:

$N^{f}$ detached $=\nu\left(\left.\varphi^{o}\right|_{\delta=0}\right)-\nu\left(\left.\varphi^{o}\right|_{\delta}\right)=\frac{\eta}{B_{2}}-\frac{\eta}{B_{2}+B_{3} \delta^{m}}$

where $\nu$ is the number of lying down monomers and $\eta$ is a constant. Substituting (7) in (13) and inserting the obtained relation in (12), we have: 

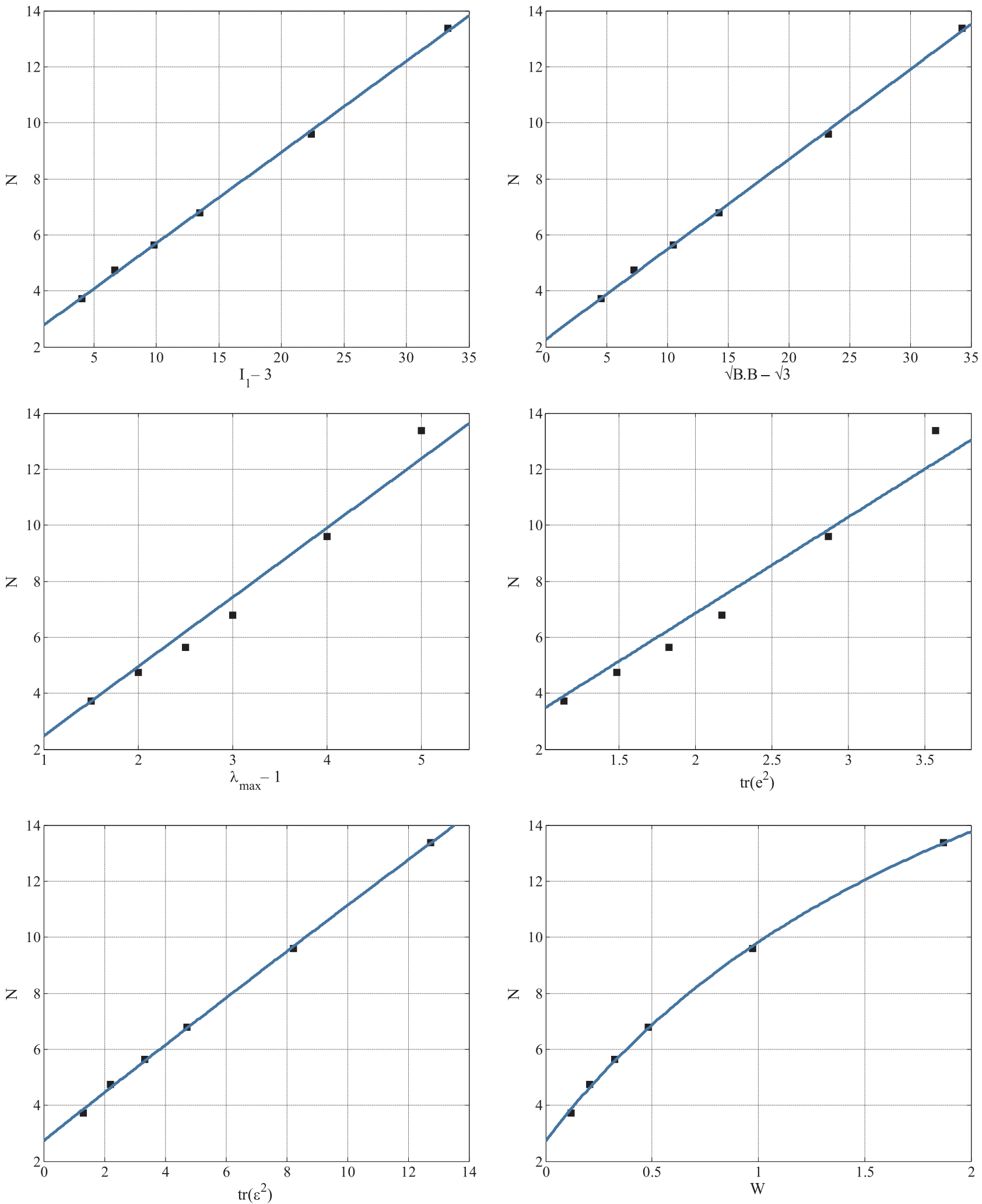

Fig. 2. Evolution of chains average length during deformation for data reported in [32]. Lines: theory, marks: experimental values.

$N=\frac{1+B_{7} \delta^{m}}{B_{8}+B_{9} \delta^{m}}$

or

$N=\frac{1+C_{6} e^{C_{1} \delta}}{C_{7}+C_{8} e^{C_{1} \delta}}$
This network alteration theory well demonstrates the evolution of network characteristic parameters and also provides a reasonable interpretation for increase in the number of active monomer segments during deformation without contravening the principle of mass conservation [25]. However, there is still an important open question; how should one define Eq. (4) and which quantity is the best choice for $\delta$ ?

Noting that definition of $\delta$ can involve quantities of any type and 
Table 2

Evolution laws, normalized RMSE's and limiting values of the network parameters for the experimental data reported by Chagnon et al. [32].

\begin{tabular}{|c|c|c|c|c|c|c|}
\hline$\delta$ & $\mu$ & NRMSE & $\lim \mu$ & $N$ & NRMSE & $\lim N$ \\
\hline$I_{1}-3$ & $\frac{1+0.01 \delta}{16.33+0.81 \delta}$ & 0.0101 & $\begin{array}{l}\delta \rightarrow 0: \mu \rightarrow 0.061 \\
\delta \rightarrow \infty: \mu \rightarrow 0.012\end{array}$ & $\frac{1+0.134 \delta}{0.41+0.14 e(-6) \delta}$ & 0.0188 & $\begin{array}{l}\delta \rightarrow 0: N \rightarrow 2.44 \\
\delta \rightarrow \infty: N \rightarrow 8.3 e 5\end{array}$ \\
\hline$\sqrt{\text { B. B }}-\sqrt{3}$ & $\frac{1+0.0096 \delta}{16.14+0.77 \delta}$ & 0.0104 & $\begin{array}{l}\delta \rightarrow 0: \mu \rightarrow 0.062 \\
\delta \rightarrow \infty: \mu \rightarrow 0.012\end{array}$ & $\frac{1+0.142 \delta}{0.44+1.72 e(-7) \delta}$ & 0.0200 & $\begin{array}{l}\delta \rightarrow 0: N \rightarrow 2.27 \\
\delta \rightarrow \infty: N \rightarrow 8.25 e 5\end{array}$ \\
\hline$\lambda_{\max }-1$ & $\frac{1+0.0066 \delta}{12.96+3.873 \delta}$ & 0.0200 & $\begin{array}{l}\delta \rightarrow 0: \mu \rightarrow 0.077 \\
\delta \rightarrow \infty: \mu \rightarrow 0.002\end{array}$ & $\frac{1+2.087 e 10 \delta}{8.43 e 9+1.36 e 5 \delta}$ & 0.1085 & $\begin{array}{l}\delta \rightarrow 0: N \rightarrow 0.00 \\
\delta \rightarrow \infty: N \rightarrow 1.53 e 5\end{array}$ \\
\hline $\operatorname{tr}\left(e^{2}\right)$ & $\frac{1+0.006 \delta}{12.46+5.5 \delta}$ & 0.0193 & $\begin{array}{l}\delta \rightarrow 0: \mu \rightarrow 0.080 \\
\delta \rightarrow \infty: \mu \rightarrow 0.001\end{array}$ & $\frac{1+2.865 e 8 \delta}{8.356 e 7+2862 \delta}$ & 0.1219 & $\begin{array}{l}\delta \rightarrow 0: N \rightarrow 0.00 \\
\delta \rightarrow \infty: N \rightarrow 1.00 e 5\end{array}$ \\
\hline $\operatorname{tr}\left(\epsilon^{2}\right)$ & $\frac{1+0.042 \delta}{16.52+2.57 \delta}$ & 0.0093 & $\begin{array}{l}\delta \rightarrow 0: \mu \rightarrow 0.060 \\
\delta \rightarrow \infty: \mu \rightarrow 0.016\end{array}$ & $\frac{1+0.317 \delta}{0.365+9.34 e(-4) \delta}$ & 0.0176 & $\begin{array}{l}\delta \rightarrow 0: N \rightarrow 2.74 \\
\delta \rightarrow \infty: N \rightarrow 339.4\end{array}$ \\
\hline$W$ & $\frac{1+0.87 \delta}{16.50+35.95 \delta}$ & 0.0089 & $\begin{array}{l}\delta \rightarrow 0: \mu \rightarrow 0.060 \\
\delta \rightarrow \infty: \mu \rightarrow 0.024\end{array}$ & $\frac{1+4.025 \delta}{0.365+0.146 \delta}$ & 0.0182 & $\begin{array}{l}\delta \rightarrow 0: N \rightarrow 2.74 \\
\delta \rightarrow \infty: N \rightarrow 27.57\end{array}$ \\
\hline
\end{tabular}
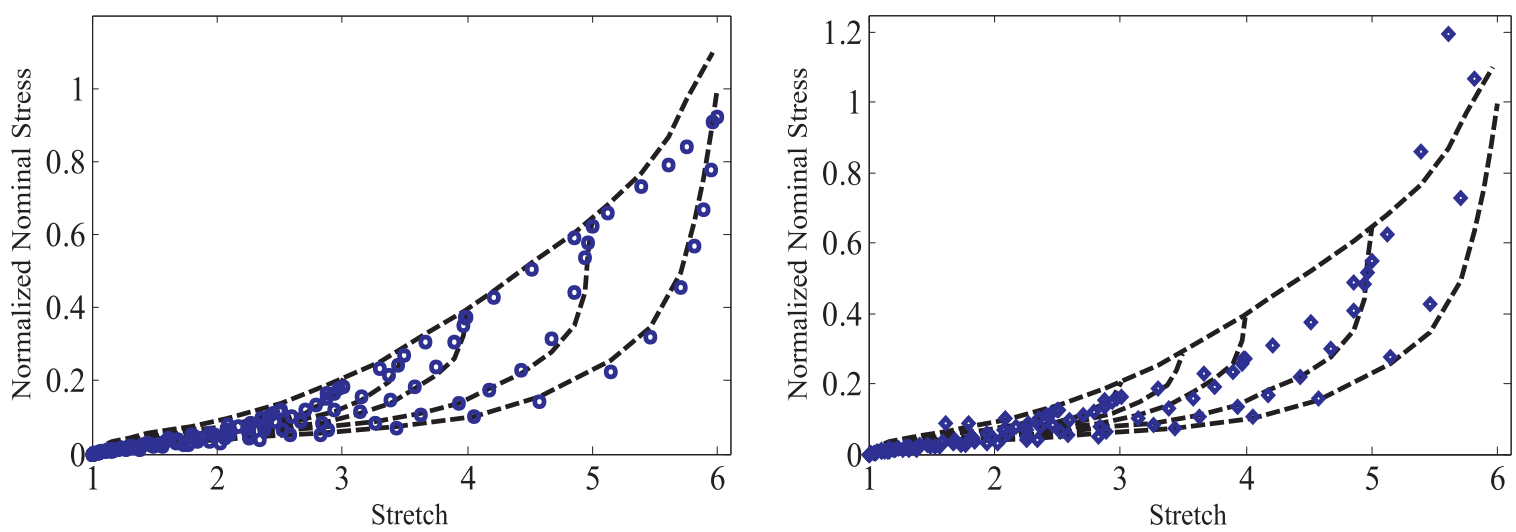

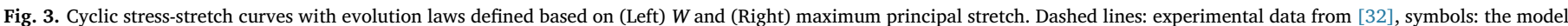
results.

order (higher-order strains, exponential, logarithmic etc.), here, in definition of (4) we only consider linear functions $(m=1)$ with respect to $\delta$ and focus on determining the best choice of $\delta$. For more investigations, one can analyze higher-order definitions $(m>1)$ as well as the exponential form.

Several quantities have been used as threshold parameter of Mullins softening in the literature, most of them as damage parameters in CDMbased models. The main purpose of the current work is to specify which quantity, of which type and which order best demonstrates the evolution of elastomers network. To this end, several measures proposed by different authors in the past years (as shown in Table 1) would be examined. It is noted that, focus of the present work is on the primary softening which occurs at the first loading. For subsequent cycles one may utilize the concept of stretch amplification [31].

\section{Results and discussion}

In this section, the measures introduced in Table 1 are utilized in conjunction with (10) and (14) to estimate the evolutions of network parameters for two sets of experiments conducted on carbon black filled natural rubber in different strain ranges.

The first data set is for tests conducted by Chagnon et al. [32] in 0$500 \%$ strain. The obtained results for normalized chain density and chains average length are shown respectively in Figs. 1 and 2 in comparison with the experiment results.

Obtained evolution laws for the first data set are summarized in Table 2. The root mean square error normalized by average of the quantity (NRMSE) is also reported in each case to compare accuracy of the obtained evolution laws. Furthermore, values of the network parameters in two limiting states are also reported; initial undeformed state (corresponding to the virgin material) and extremely high-strain state. Though due to material failure the latter state is not achieved in reality, however, the limiting values in this state can also be illustrative. From (10) and (14) we have:

$$
\begin{array}{rlrl}
\delta & =0: & \mu & =\left(1-\phi_{f}\right) \mu^{\text {gum }}+\phi_{f} \mu_{0}{ }^{f}=\frac{1}{B_{5}} \\
\delta \rightarrow \infty: & \lim \mu & =\left(1-\phi_{f}\right) \mu^{g u m}=\frac{B_{4}}{B_{6}}
\end{array}
$$

$$
\begin{array}{rlrl}
\delta & =0: & N & =\frac{1}{B_{8}} \\
\delta \rightarrow \infty: & \lim N & =\frac{B_{7}}{B_{9}}
\end{array}
$$

From Figs. 1 and 2 one concludes that, the fractional laws based on Langmuir theory provide an efficient framework for evolution of 

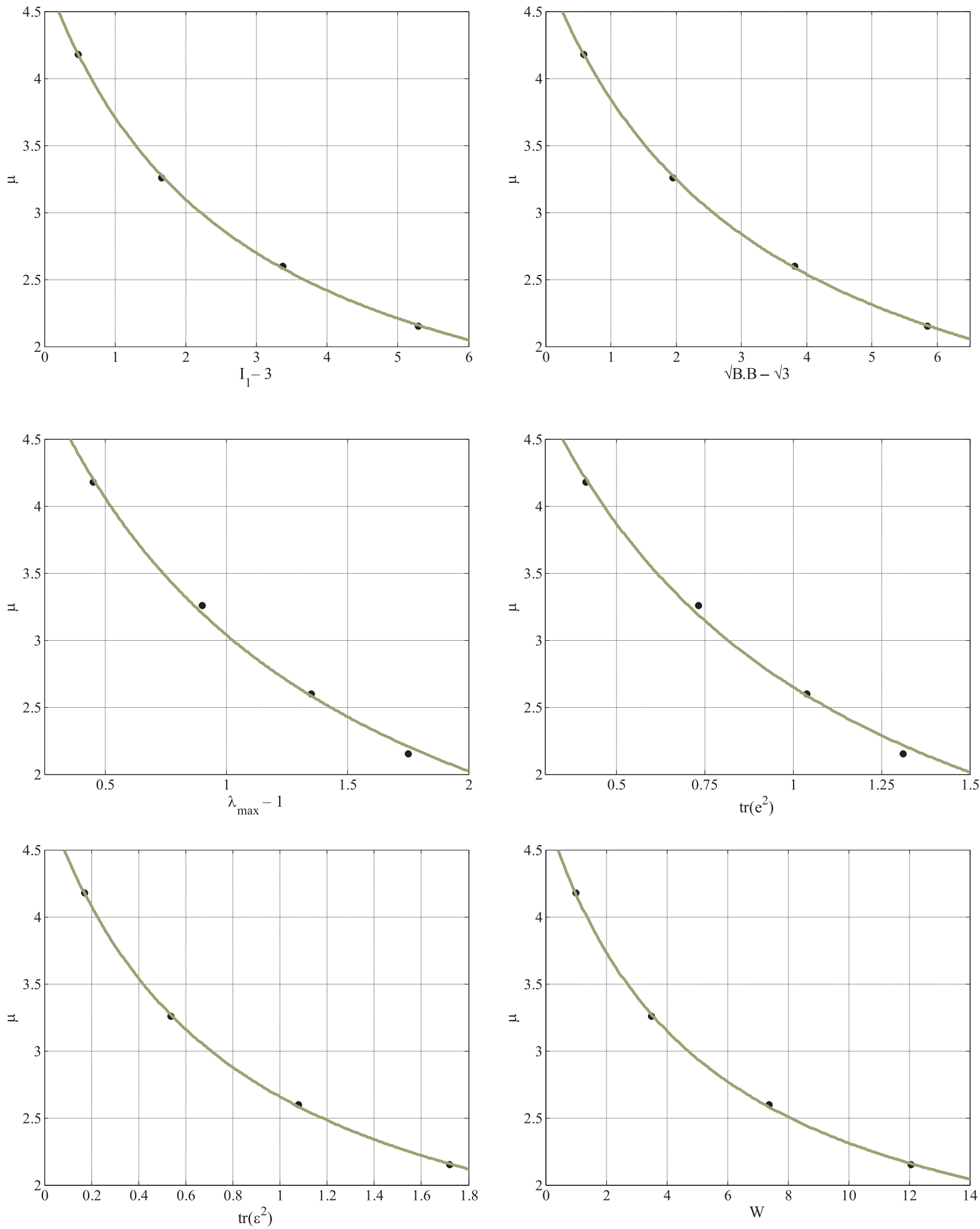

Fig. 4. Evolution of network chain density during deformation for data reported in [10]. Lines: theory, marks: experimental values.

rubbers molecular network. According to Table 2, $I_{1}-3, \sqrt{\mathrm{B} . \mathrm{B}}-\sqrt{3}$, Piola strain and $W$ give more accurate fittings for $\mu$. These parameters also have similar predictions for the limiting values of $\mu$. One should notice that, the remaining parameters do not give either good fittings or reasonable values for limiting states.
Regarding the chains average length, $I_{1}-3, \sqrt{\mathrm{B} . \mathrm{B}}-\sqrt{3}$, Piola strain and $W$ provide acceptable fittings but investigation of the limiting values reveals only $W$ gives reasonable values for the limiting states. Accordingly, one may conclude that, only $W$ can be used as a reliable parameter in predicting the Mullins effect for this material. Many strain 

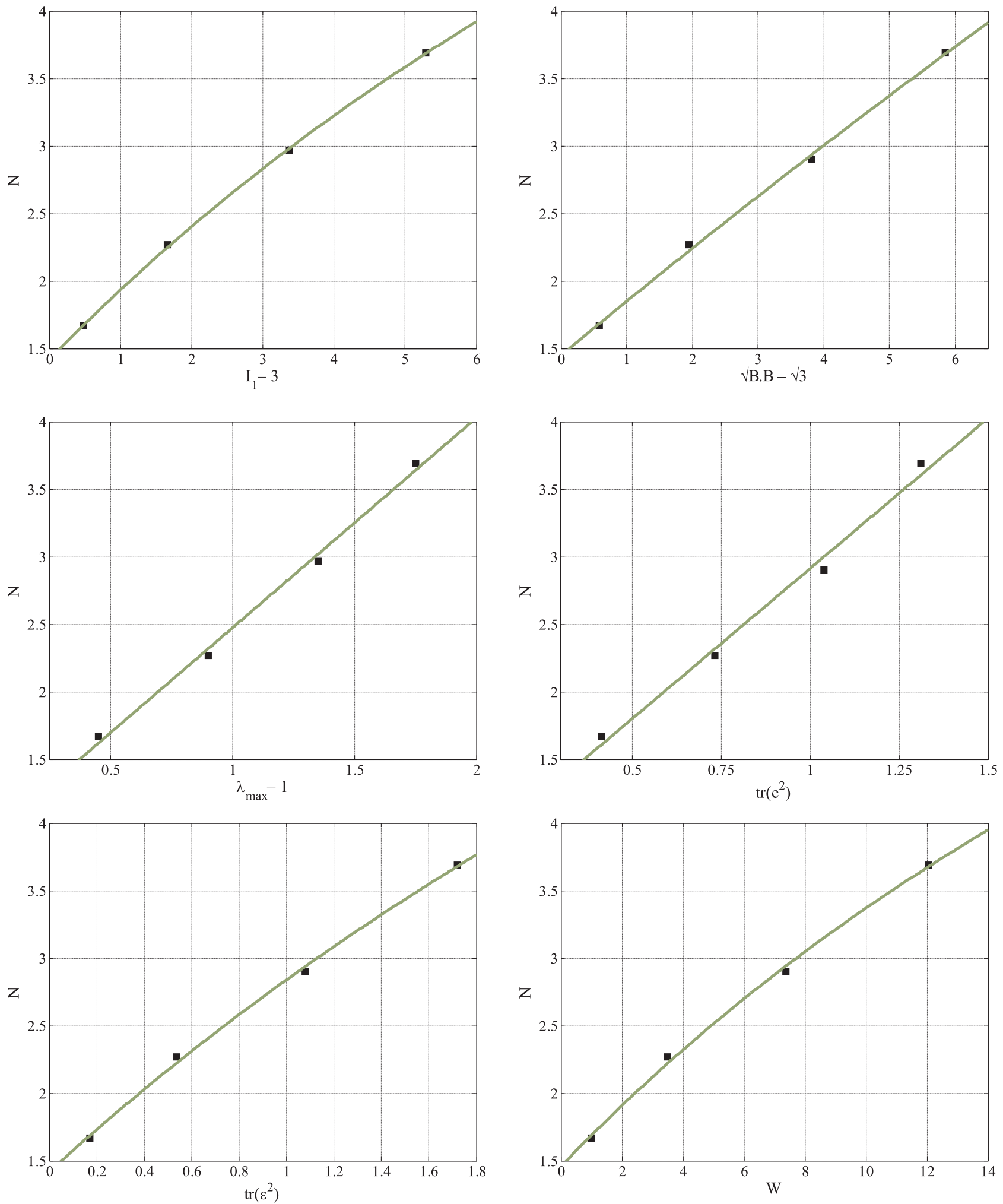

Fig. 5. Evolution of chains average length during deformation for data reported in [10]. Lines: theory, marks: experimental values.

energy functions have been proposed in the literature for rubber-like materials but one should choose an appropriate one to have reasonable results even in other modes of deformation [35-38]. Using the Gent model [39] which explicitly includes the network parameters, along with the evolution laws defined based on the stored strain energy the stress-stretch relation for uniaxial deformation is obtained as:
$f=\mu(\overparen{W}) \frac{J_{m}(\overparen{W})}{J_{m}(\widehat{W})-\left(I_{1}-3\right)}\left(\lambda-\lambda^{-2}\right) \quad$ where

$J_{m}(\overparen{W})=3[N(\overparen{W})-1], \quad \widehat{W}=\max \underset{0 \leq \tau \leq t}{W(\tau)}$

In relation (18), $f$ is the nominal stress. Also regarding Table 2, $\mu(\overparen{W})$ and $N(\overparen{W})$ are defined as follows: 
Table 3

Evolution laws, normalized RMSE's and limiting values of the network parameters for the experimental data reported by Diani et al. [10].

\begin{tabular}{|c|c|c|c|c|c|c|}
\hline$\delta$ & $\mu$ & NRMSE & $\lim \mu$ & $N$ & NRMSE & $\lim N$ \\
\hline$I_{1}-3$ & $\frac{1+0.062 \delta}{0.21+0.077 \delta}$ & 0.0077 & $\begin{array}{l}\delta \rightarrow 0: \mu \rightarrow 4.764 \\
\delta \rightarrow \infty: \mu \rightarrow 0.805\end{array}$ & $\frac{1+0.432 \delta}{0.706+0.035 \delta}$ & 0.0092 & $\begin{array}{l}\delta \rightarrow 0: N \rightarrow 1.416 \\
\delta \rightarrow \infty: N \rightarrow 12.34\end{array}$ \\
\hline$\sqrt{\mathrm{B} \cdot \mathrm{B}}-\sqrt{3}$ & $\frac{1+0.039 \delta}{0.209+0.062 \delta}$ & 0.0061 & $\begin{array}{l}\delta \rightarrow 0: \mu \rightarrow 4.785 \\
\delta \rightarrow \infty: \mu \rightarrow 0.629\end{array}$ & $\frac{1+0.356 \delta}{0.711+0.0215 \delta}$ & 0.0228 & $\begin{array}{l}\delta \rightarrow 0: N \rightarrow 1.406 \\
\delta \rightarrow \infty: N \rightarrow 16.56\end{array}$ \\
\hline$\lambda_{\max }-1$ & $\frac{1+3.7 e(-15) \delta}{0.163+0.166 \delta}$ & 0.0200 & $\begin{array}{l}\delta \rightarrow 0: \mu \rightarrow 6.135 \\
\delta \rightarrow \infty: \mu \rightarrow 0.000\end{array}$ & $\frac{1+1.676 \delta}{1.09+1.02 e(-4) \delta}$ & 0.0376 & $\begin{array}{l}\delta \rightarrow 0: N \rightarrow 0.917 \\
\delta \rightarrow \infty: N \rightarrow 16431\end{array}$ \\
\hline $\operatorname{tr}\left(e^{2}\right)$ & $\frac{1+4.9 e(-10) \delta}{0.139+0.238 \delta}$ & 0.0235 & $\begin{array}{l}\delta \rightarrow 0: \mu \rightarrow 7.194 \\
\delta \rightarrow \infty: \mu \rightarrow 0.000\end{array}$ & $\frac{1+3.303 \delta}{1.467+2.6 e(-4) \delta}$ & 0.0572 & $\begin{array}{l}\delta \rightarrow 0: N \rightarrow 0.681 \\
\delta \rightarrow \infty: N \rightarrow 12692\end{array}$ \\
\hline $\operatorname{tr}\left(\epsilon^{2}\right)$ & $\frac{1+0.243 \delta}{0.205+0.263 \delta}$ & 0.0069 & $\begin{array}{l}\delta \rightarrow 0: \mu \rightarrow 4.878 \\
\delta \rightarrow \infty: \mu \rightarrow 0.924\end{array}$ & $\frac{1+1.532 \delta}{0.729+0.150 \delta}$ & 0.0237 & $\begin{array}{l}\delta \rightarrow 0: N \rightarrow 1.372 \\
\delta \rightarrow \infty: N \rightarrow 10.213\end{array}$ \\
\hline$W$ & $\frac{1+0.041 \delta}{0.21+0.04 \delta}$ & 0.0078 & $\begin{array}{l}\delta \rightarrow 0: \mu \rightarrow 4.761 \\
\delta \rightarrow \infty: \mu \rightarrow 1.025\end{array}$ & $\frac{1+0.222 \delta}{0.705+0.024 \delta}$ & 0.0248 & $\begin{array}{l}\delta \rightarrow 0: N \rightarrow 1.418 \\
\delta \rightarrow \infty: N \rightarrow 9.251\end{array}$ \\
\hline
\end{tabular}
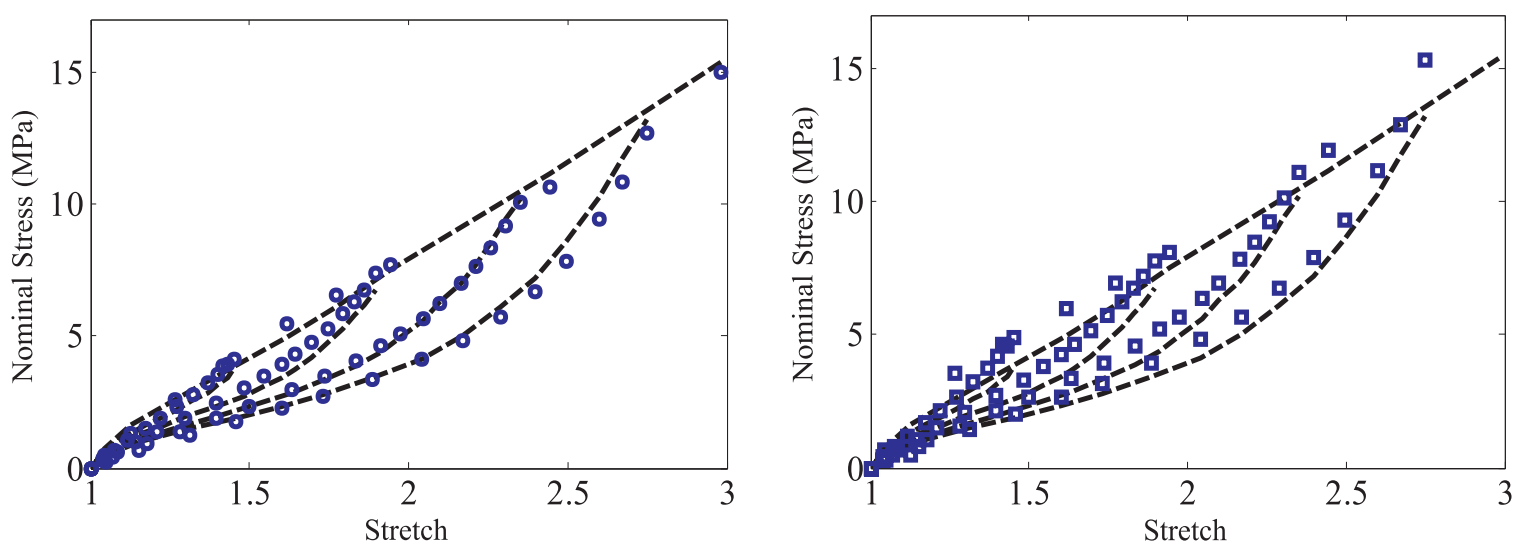

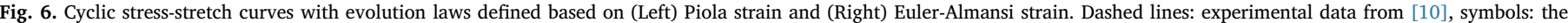
model results.

$\mu(\overparen{W})=\frac{1+0.87 \overparen{W}}{16.5+35.95 \overparen{W}}, \quad \quad N(\widehat{W})=\frac{1+4.025 \overparen{W}}{0.365+0.146 \overparen{W}}$

Cyclic stress-stretch curves are reproduced using the evolutions of the network parameters defined based on the strain energy function and the maximum principal stretch. Comparisons of the reproduced cyclic curves with experimental data are shown in Fig. 3. It is seen that, the evolutions defined based on the strain energy function provide more accurate results for the first loading and also the subsequent loadingunloading curves.

The second data set has been reported by Diani et al. [10] in 0$200 \%$ strain range. Estimated evolutions are shown in Figs. 4 and 5 for chain density and chains average length in comparison with the test results. The evolution laws are also summarized in Table 3 along with normalized root mean square errors and the limiting values of the network parameters.

According to Table $3, I_{1}-3, \sqrt{\mathrm{B} . \mathrm{B}}-\sqrt{3}$, Piola strain and $W$ are relatively good choices for predicting the evolution of chains density for this data. These parameters also provide near and reasonable estimations for $\mu$ at limiting states. The same parameters give satisfactory results for evolution of chains average length (note that $I_{1}-3$ is more accurate among others). Consequently, $I_{1}-3, \sqrt{\mathrm{B} . \mathrm{B}}-\sqrt{3}$, Piola strain and $W$ seem to be good thresholds of Mullin softening for this data.
This time, comparisons of the experimental cyclic stress-stretch curves are presented with those reproduced based on Piola and EulerAlmansi strains. As shown in Fig. 6, the evolution laws defined based on Piola strain are in good agreement with experimental data but the curves based on Euler-Almansi strain are not so reliable especially at first loading and also unloading from small stretches because this evolution parameter does not provide reasonable limiting values for the network parameters.

Multiplication of the evolutions for chains density and average length obtained from each measure gives the evolution of active monomer segments with respect to that measure. Comparisons of the predictions with experimental data are presented in Figs. 7 and 8 for both data sets, respectively. Also, the normalized RMSE for each evolution law is shown in Tables 4 and 5 for both data sets. For the first data, the stored strain energy exhibits an accurate fitting where $I_{1}-3, \sqrt{\text { B.B }}-\sqrt{3}$ and Piola strain are also accurate for second data in addition to the strain energy.

\section{Summary and conclusions}

The network alteration theory recently proposed by the author was extended to a more general form. The evolution of rubbers molecular network during deformation was defined based on different measures to 

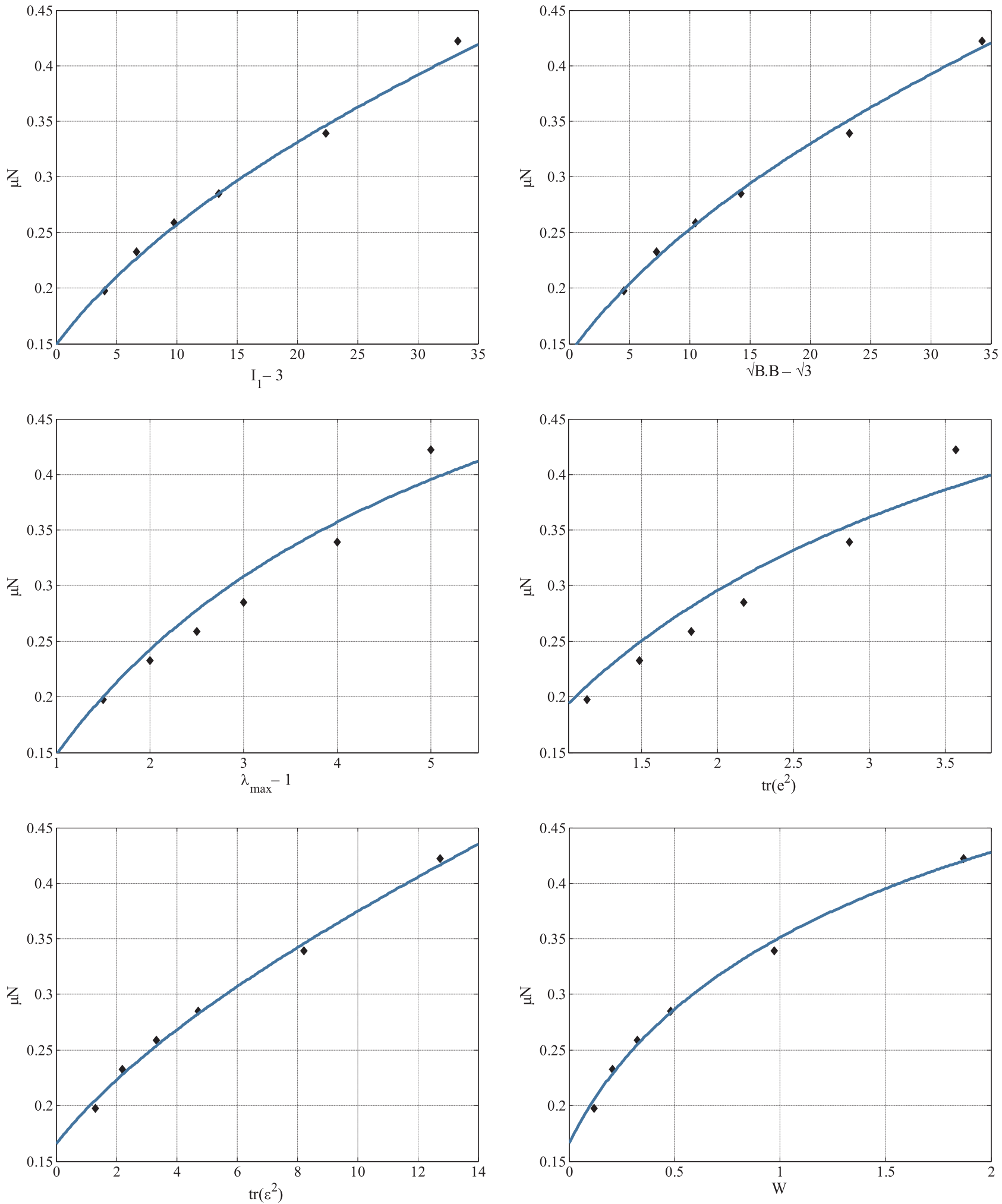

Fig. 7. Evolution of active monomers during deformation for data reported in [32]. Lines: theory, marks: experimental values.

evaluate efficiency of each measure in predicting the Mullins softening. Two experimental data sets in different strain ranges were thoroughly investigated to determine a reliable threshold for the observed Mullins softening in natural rubber.

It was concluded that, maximum principal stretch and the EulerAlmansi strain are not good thresholds of Mullins effect at all. Other investigated measures are almost efficient at small stretches but they mostly become less and less accurate at large stretches. However, the stored strain energy preserves its efficiency at whole stretch range. A reasonable reason for good efficiency of this measure may be that it involves the amount of applied stress in addition to the strain. It implies that, investigations on efficiency of different stress measures in context of network alteration theories can be subject of future works. 

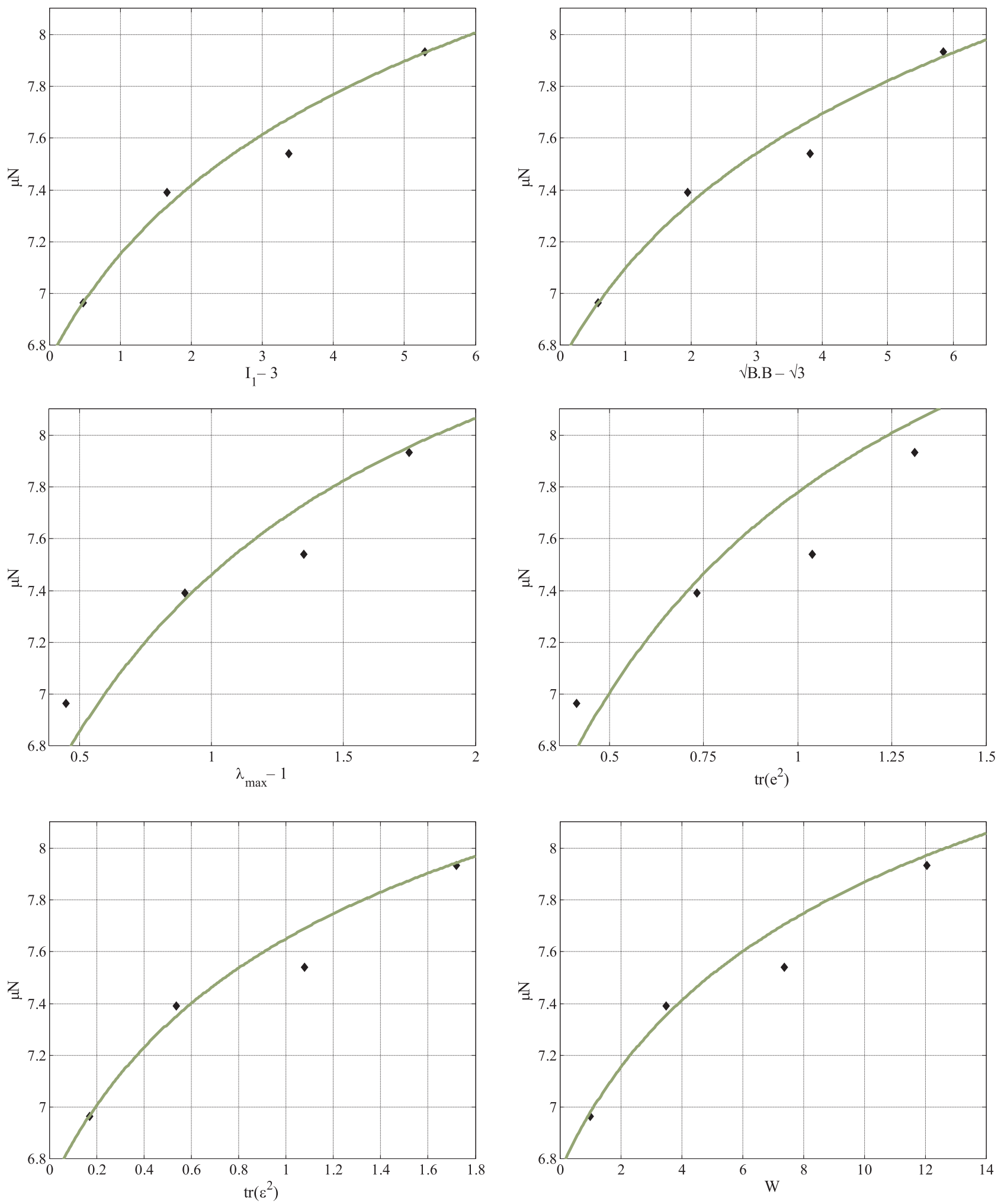

Fig. 8. Evolution of active monomers during deformation for data reported in [10]. Lines: theory, marks: experimental values.

Table 4

Normalized RMSE's of the number of active monomers for the experimental data reported by Chagnon et al. [32].

\begin{tabular}{lllllll}
\hline$\delta$ & $I_{1}-3$ & $\sqrt{\mathrm{B} . \mathrm{B}}-\sqrt{3}$ & $\lambda_{\max }-1$ & $\operatorname{tr}\left(e^{2}\right)$ & $\operatorname{tr}\left(\epsilon^{2}\right)$ & $W$ \\
NRMSE & 0.0242 & 0.0228 & 0.0696 & 0.0819 & 0.0207 & 0.0211 \\
\hline
\end{tabular}

Table 5

Normalized RMSE's of the number of active monomers for the experimental data reported by Diani et al. [10].

\begin{tabular}{lllllll}
\hline$\delta$ & $I_{1}-3$ & $\sqrt{\mathrm{B} . \mathrm{B}}-\sqrt{3}$ & $\lambda_{\max }-1$ & $\operatorname{tr}\left(e^{2}\right)$ & $\operatorname{tr}\left(\epsilon^{2}\right)$ & $W$ \\
NRMSE & 0.0111 & 0.0092 & 0.0209 & 0.0235 & 0.0119 & 0.0115 \\
\hline
\end{tabular}




\section{Acknowledgements}

The author thanks the Iran National Science Foundation (INSF) for supporting this work.

\section{References}

[1] Mullins L. Effect of Stretching On The Properties Of Rubber. Rubber Chem Technol 1948;21(2):281-300.

[2] Morozov IA, Komar LA, Lauke B. Structural-mechanical model of filled rubber: influence of filler arrangement. Int J Mech Sci 2016;107:160-9.

[3] Luo Y, Liu Y, Yin HP. Numerical investigation of nonlinear properties of a rubber absorber in rail fastening systems. Int J Mech Sci 2013;69:107-13.

[4] Khajehsaeid H, Baghani M, Naghdabadi R. Finite strain numerical analysis of elastomeric bushings under multi-axial loadings: a compressible visco-hyperelastic approach. Int J Mech Mater Des 2013:1-15.

[5] Tomita Y, Azuma K, Naito M. Computational evaluation of strain-rate-dependent deformation behavior of rubber and carbon-black-filled rubber under monotonic and cyclic straining. Int J Mech Sci 2008;50(5):856-68.

[6] Khajehsaeid H, Ramezani MA. Visco-hyperelastic modeling of automotive elastomeric bushings with emphasis on the coupling effect of axial and torsional deformations. In: Society of Engineering Science Proceedings of the 51st Annual Technical Meeting. West Lafayette, Purdue University Purdue University Libraries Scholarly Publishing Services; 2014.

[7] Khajehsaeid H. Modeling nonlinear viscoelastic behavior of elastomers using a micromechanically motivated rate-dependent approach for relaxation times involved in integral-based models. Const Models Rubber IX 2015:165-70.

[8] Kachanov L. Time of the rupture process under creep conditions. Isv Akad Nauk Ssr Otd Tekh Nauk 1958;8:26-31.

[9] Kaliske M, Nasdala L, Rothert H. On damage modelling for elastic and viscoelastic materials at large strain. Comput Struct 2001;79(22-25):2133-41.

[10] Diani J, Brieu M, Vacherand JM. A damage directional constitutive model for Mullins effect with permanent set and induced anisotropy. Eur J Mech - A/Solids 2006;25(3):483-96.

[11] De Tommasi D, Puglisi G, Saccomandi G. A micromechanics-based model for the Mullins effect. J Rheol 2006;50(4):495-512.

[12] De Tommasi D, et al. Damage and healing effects in rubber-like balloons. Int J Solids Struct 2009;46(22):3999-4005.

[13] Laiarinandrasana L, Piques R, Robisson A. Visco-hyperelastic model with internal state variable coupled with discontinuous damage concept under total Lagrangian formulation. Int J Plast 2003;19(7):977-1000.

[14] Lion A. A constitutive model for carbon black filled rubber: experimental investigations and mathematical representation. Contin Mech Thermodyn 1996;8(3):153-69.

[15] Dorfmann A, Ogden RW. A constitutive model for the Mullins effect with permanent set in particle-reinforced rubber. Int J Solids Struct 2004;41(7):1855-78.

[16] Horgan CO, Ogden RW, Saccomandi G. A theory of stress softening of elastomer based on finite chain extensibility. Proceedings of the Royal Society of London. Series A: Mathematical, Physical and Engineering Sciences. 460(2046): p. 17371754; 2004.

[17] Ogden RW, Roxburgh DG. A pseudo-elastic model for the Mullins effect in filled rubber. Proceedings of the Royal Society of London A: Mathematical, Physical and Engineering Sciences, 1999. 455: p. 2861-2877; 1988.

[18] Machado G, Chagnon G, Favier D. Analysis of the isotropic models of the Mullins effect based on filled silicone rubber experimental results. Mech Mater 2010;42(9):841-51.

[19] Papkov V, et al. Energy investigation of the softening of siloxane rubbers during deformation. Polym Mech 1975;11(3):329-33.

[20] Hanson DE, et al. Stress softening experiments in silica-filled polydimethylsiloxane provide insight into a mechanism for the Mullins effect. Polymer 2005;46(24):10989-95.

[21] Fukahori Y. The mechanics and mechanism of the carbon black reinforcement of elastomers. Rubber Chem Technol 2003;76(2):548-66.

[22] Diani J, Fayolle B, Gilormini P. A review on the Mullins effect. Eur Polym J 2009;45(3):601-12.

[23] De Tommasi D, Puglisi G, Saccomandi G. Multiscale mechanics of macromolecular materials with unfolding domains. J Mech Phys Solids 2015;78:154-72.

[24] Klüppel M, Schramm J. A generalized tube model of rubber elasticity and stress softening of filler reinforced elastomer systems. Macromol Theory Simul 2000;9(9):742-54.

[25] Khajehsaeid H. Development of a network alteration theory for the Mullinssoftening of filled elastomers based on the morphology of filler-chain interactions. Int J Solids Struct 2016;80:158-67.

[26] Chagnon G, et al. Development of new constitutive equations for the Mullins effect in rubber using the network alteration theory. Int J Solids Struct 2006;43(2223):6817-31.

[27] Marckmann G, et al. A theory of network alteration for the Mullins effect. J Mech Phys Solids 2002;50(9):2011-28.

[28] Maier P, Goritz D. Molecular interpretation of the Payne effect. Kautsch Gummi Kunstst 1996;49(1):18-21.

[29] Edwards S, Vilgis TA. The tube model theory of rubber elasticity. Rep Prog Phys 1988;51(2):243-59.

[30] Khajehsaeid H, et al. Strain and stress concentrations in elastomers at finite deformations: effects of strain-induced crystallization, filler reinforcement, and deformation rate. Acta Mech 2016;227(7):1969-82.

[31] Merckel Y, et al. Experimental characterization and modelling of the cyclic softening of carbon-black filled rubbers. Mater Sci Eng: A 2011;528(29):8651-9.

[32] Chagnon G, et al. On the relevance of continuum damage mechanics as applied to the Mullins effect in elastomers. J Mech Phys Solids 2004;52(7):1627-50.

[33] Zúñiga EA. A phenomenological energy-based model to characterize stress-softening effect in elastomers. Polymer 2005;46(10):3496-506.

[34] Krishnaswamy S, Beatty MF. The Mullins effect in compressible solids. Int J Eng Sci 2000;38(13):1397-414.

[35] Khajehsaeid H, et al. A visco-hyperelastic constitutive model for rubber-like materials: a rate-dependent relaxation time scheme. Int J Eng Sci 2014;79(0):44-58.

[36] Khajehsaeid H, Arghavani J, Naghdabadi R. A hyperelastic constitutive model for rubber-like materials. Eur J Mech - A/Solids 2013;38(0):144-51.

[37] Boyce MC, Arruda EM. Constitutive models of rubber elasticity: a review. Rubber Chem Technol 2000;73:505-23.

[38] Khajehsaeid H, Naghdabadi R, Arghavani J. A strain energy function for rubber-like materials. Const Models Rubber 2013;8:205-10.

[39] Gent A. A new constitutive relation for rubber. Rubber Chem Technol 1996;69:59-61. 